\title{
Seeing is Deceiving: The Psychology and Neuroscience of Fake Faces
}

\author{
Sujal Manohar, BS, BA \\ Duke University
}

Address for Correspondence:

Sujal Manohar

Duke University

sujal.manohar@duke.edu

ORCID iD: 0000-0001-7128-7043 


\begin{abstract}
$\underline{\text { Abstract }}$
Face perception is an essential skill for human interaction and social learning, impacting how we build relationships and perceive the world around us. In the modern era, face processing remains important but confronts new challenges due to recent technological advances such as deepfake technology and AI-generated faces. These computer-generated (CG) faces may be difficult for our brains to distinguish from real faces, raising questions in the fields of criminal justice, politics, and animation, to name a few. This review explores the neurobiology of face processing and its interplay with affect, laying the foundation for an investigation into recent studies examining how humans differentiate between real and CG faces. Studies on the uncanny valley effect and pareidolia offer further insights as to how humans make this judgment and the possible boundaries of face perception. Additional research is needed to better understand this emerging area and possibly train human viewers to perform these judgments more accurately in the future.
\end{abstract}

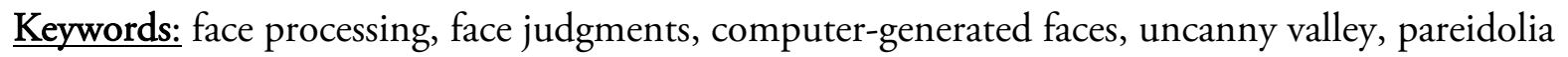




\section{Introduction}

At a May 2019 news conference, Speaker of the House Nancy Pelosi stammered when speaking to the press, unable to communicate clearly. A usually polished speaker, she slurred her words and appeared slow, mentioning three problems while only holding up two fingers. Her critics pointed to the video as evidence of her senility or a possible mental health problem, indicating she was not fit for her job (Venkataramakrishnan, 2019). However, the video footage of this event was not real-it was a prime example of "deepfake" technology, AI-generated content designed to fool humans (Venkataramakrishnan, 2019). Yet humans, being visual creatures, believed what they saw. Where do we draw the line between the real and the fake? More importantly, how does a human brain, evolved in a world where such visual events could not be faked, operate in a world where they can? This brings up questions of how we normally process face stimuli and how to differentiate between real and computer generated (CG) images in a world with rapidly advancing technology.

As CG faces become increasingly lifelike, the rapid rise of fake face data will create new issues for society, particularly in the fields of politics, criminal justice, psychology, and human-computer interaction. Photographs are core pieces of evidence in many criminal trials, whether footage from a security camera or pornographic images from a suspect's computer. Research on the uncanny valley, the uncomfortable feeling viewers get from almost lifelike CG faces, also suggests important implications for the fields of virtual reality and animation (MacDorman et al., 2009, Mori et al., 2012). Previously, creating lifelike images required highly paid professionals using professional-grade computer graphics software; now, widely available neural network methods enable anyone to generate huge volumes of fake data for audio, video, text, images, and more. After the development of Generative Adversarial Networks (GANs), fake face images could be created more easily and rapidly (Goodfellow et al., 2014). Sophisticated algorithms perform style transfers, even editing photographs to represent the styles of popular paintings (Gatys et al., 2016). While new computer algorithms can detect artificial faces and doctored images (Korshunov \& Marcel, 2019, DangNguyen et al., 2012, Farid, 2006, L. Jiang et al., 2020), humans perform this task very differently. Therefore, it is critical to determine how we differentiate between real and CG images.

All of these issues are symptomatic of the fact that face processing, a core human skill evolutionarily molded for thousands of years, has not evolved to counter the challenge posed by CG faces. And though fake news text (W. Y. Wang, 2017), artificial voice impersonation (Mukhopadhyay et al., 2015), and forged videos (Rössler et al., 2018) are also pertinent to a modern context, this review will focus on CG face images. Faces pose a particular challenge because humans interact with the world through vision and face-to-face communication, skills also involved in decision-making and judgment. In the past, facial expressions promoted social learning and coordination, likely giving insight into behavioral intentions (Eisenbarth \& Alpers, 2011). Humans still depend on visual inputs in the modern era, but while face perception remains essential, we must now process unfamiliar types of stimuli.

Since most previous work has investigated judgments about faces in normal circumstances, comparatively less is known about how we determine whether face images are real. In this review, we will explore the development of the neurobiology and psychology of face processing, as well as its 
interplay with affect. We will also summarize recent research on how humans make these face-related judgments, and how this process may change in light of new technologies. We will then show how phenomena such as the uncanny valley and pareidolia define the boundaries of facial perception and judgment. The review concludes by offering new directions for future research.

\section{Neurobiology of Face Processing}

Facial recognition is an instantaneous and robust process for humans, occurring just 70 milliseconds after stimulus presentation (Nemrodov et al., 2016). Likewise, implicit trait judgments, such as trustworthiness and aggressiveness, are made incredibly quickly, often within the first 100 milliseconds (Willis \& Todorov, 2006). There is also evidence these distinctive face processing mechanisms are present from a very young age; newborn infants preferentially track faces compared to other stimuli from the age of two months (Collins \& Olson, 2014), and preference for same-race faces emerges at three months (S. Liu et al., 2011). Face recognition depends on information from three-dimensional shapes and two-dimensional surface reflectance (F. Jiang et al., 2011) and studies with human participants confirm a shape-based model of face processing (Riesenhuber et al., 2004). Facial recognition skills emerge at an early age and facial judgments occur rapidly, suggesting the importance of face processing in human interaction and survival. Since reliable face processing is a core part of how humans, a visual and social species, engage with the world, it is thus not surprising that face processing is fast and under strong developmental control.

Importantly, the behavioral importance of face processing is matched by a dedicated network of structures in the brain. Early studies of facial specialization began in the 1990s with the demonstration by Charlie Gross of the existence of a series of networks and regions likely involved in face processing (Gross et al., 1992). Gross discovered cells in the inferior temporal cortex that responded selectively to faces and hands, confirming earlier ideas about "grandmother cells," which only react to highly specific, meaningful stimuli (Gross, 2002). Functional magnetic resonance imaging (fMRI) experiments in humans identified an area in the fusiform gyrus that is selective for the perception of faces (Kanwisher et al., 1997). Further fMRI studies in macaques found a faceselective cortical region, and single-cell recordings confirmed this result (Tsao et al., 2006, Kriegeskorte et al., 2007), revealing six patches in the macaque inferior temporal lobe that responded to faces. Stimulating one of these patches activated the others (Moeller et al., 2008). Macaque faceselective cortical regions also corresponded closely to human brain areas (Tsao et al., 2008). These studies all indicate that the brain devotes specialized resources to recognizing and processing faces. While a few discrete sites are key for facial processing, different parts of this process may occur in a larger network of areas in the brain (Schrouff et al., 2020).

In a series of papers on human face processing, these perspectives were subsequently merged. Non-invasive studies in humans showed that individual faces elicited unique response patterns in the anterior temporal cortex (Kriegeskorte et al., 2007). Recordings from human cells confirmed this idea of hierarchical processing, since neural pathways in the medial temporal lobe create increasingly specific representations of visual stimuli over time (Mormann et al., 2008). Other studies suggested that face information is not only distributed across anatomical areas, but also across time (Schrouff et al., 2020). Furthermore, when viewing face stimuli, neurons with a higher response latency were also 
more selective (Mormann et al., 2008). Over half of neurons in the amygdala are responsive to faces, and often are more responsive when shown a small portion of the face rather than the face in entirety (Rutishauser et al., 2011). The medial temporal lobe also likely represents different categories of visual stimuli (Kreiman et al., 2000), and in some cases, certain neurons in this region were selectively activated by a particular face, like Halle Berry (Quiroga et al., 2005).

Other studies indicate that a network of anterior temporal and fusiform areas are involved in individual face recognition (Nestor et al., 2011), and the ventral anterior temporal lobes also may play a centralized role (Collins \& Olson, 2014). Electrical stimulation of the posterior fusiform site (pFUS) led to distortion of conscious face processing in human subjects, which offers further support for the role of the fusiform area (Schrouff et al., 2020). Another recent study sheds light on how all of these face regions interact (Y. Wang et al., 2020). Through diffusion MRI and mapping of the connectome, three face processing pathways were identified: one focused on static features, another that processes dynamic information, and another involved in social and emotional components of faces (Y. Wang et al., 2020). Multiple neural structures connected across different parts of the brain are involved in recognizing and responding to faces, indicating their importance for human interaction and survival.

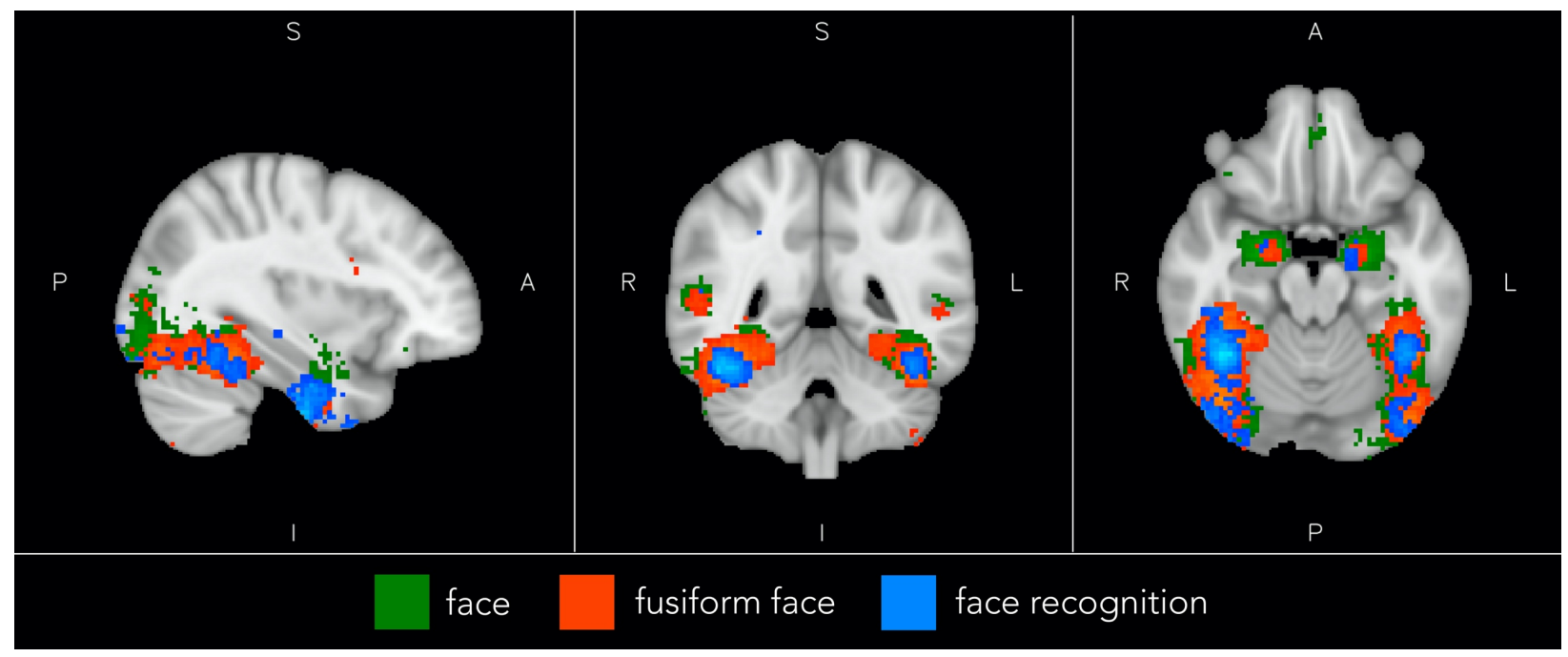

Figure 1. Consensus reverse inference facial network. Union of coordinates returned from NeuroSynth (neurosynth.org) using the search terms "face," "fusiform face," and "face recognition" (Yarkoni et al., 2011).

Taken together, all of these studies establish that visual animals like humans and monkeys have evolved specialized "hardware" to make sense of faces, but this alone doesn't tell the whole story of face processing. Simply recognizing a face is not enough — we must map it to affective and contextual information for complete processing. 


\section{$\underline{\text { Affective Face Processing }}$}

In 1923, a 53-year-old French woman genuinely believed everyone around her had been replaced by impostors. She thought she was a wealthy royal, and that her children who had passed away years earlier were actually abducted (Chhaya, 2017). Psychiatrist Joseph Capgras first described this condition where individuals believe they are surrounded by impostors. Now known as Capgras Syndrome, it remains rare and tied to other neurologic and psychiatric problems (Chhaya, 2017). In some cases, this phenomenon is modality specific: one patient believed his parents were impostors when seeing them in person but not on the phone (Hirstein \& Ramachandran, 1997). Patients with Capgras Syndrome have no differential responding to familiar compared to unfamiliar faces, and also have lowered autonomic responses to face stimuli (Ellis et al., 1997). This shows that perception is more than just recognizing a face is present-it must also bind to affective information, such as context and memory, to determine if a face is a real identity rather than a fake or impostor.

This affective information often comes in the form of factors that identify individuals within groups. Past studies showed that information from faces, such as the implicit traits trustworthiness and aggressiveness, is processed and judged rapidly with little effort ("Mapping the Social Space of the Face," 2010, Willis \& Todorov, 2006). Magnetoencephalography experiments suggested that the gender and age of a face are processed even before we identify it (Dobs et al., 2019). Further studies demonstrated the impact of age (Gunning-Dixon et al., 2003), gender (Proverbio et al., 2006), and experience (Golarai et al., 2017) on affective processing.

Facial expressions are also key pieces of affective information which impact face perception and the processing of facial identity (Vuilleumier \& Pourtois, 2007). Furthermore, previous findings describe distinctive eye movement patterns when judging genuine versus fake smiles (Calvo et al., 2013) and varying fixation patterns when looking at faces with different emotions (Schurgin et al., 2014, Wells et al., 2016). While holistic emotion recognition relies on visual, voice, and touch information (Schirmer \& Adolphs, 2017), the perception of emotions especially depends on viewing the eye and mouth regions (Eisenbarth \& Alpers, 2011). These factors, which differentiate individuals within larger groups and offer insight into emotional state, provide essential components of overall facial perception. As the case of Capgras Syndrome indicates, these dimensions are just as important as raw visual processing for normal human perception and facial recognition.

Social context is also important affective information that impacts how we view faces, as decoding facial affect is critical for social interaction and adaptation (Thomas et al., 2007). Previously viewed faces, viewing context, and even cultural background determine how faces are perceived (Webster \& MacLeod, 2011, Kelly et al., 2011, (Blais et al., 2008). Social neuroscience suggests the medial pre-frontal cortex (mPFC) is critical for social cognition, but outgroups that elicit disgust result in a lowered mPFC response (Harris \& Fiske, 2007). The lowest outgroups activated the insula and amygdala, associated with anger and disgust, rather than the mPFC, indicating the process underlying the dehumanization of other groups (Harris \& Fiske, 2006). fMRI, electrical neuroimaging, and eye tracking data all support the idea that emotional processing impacts neural systems involved in face recognition and memory (Vuilleumier \& Pourtois, 2007). It is well understood that specialized neural areas are involved in face processing; however, it is clear 
that simply recognizing a stimulus as a face is not enough — normal cognition involves integrating affective components, not just one or two pieces. Now, we will look at conditions where the normal functioning of these systems is hijacked by unusual stimuli: CG faces.

\section{The Uncanny Valley, Pareidolia, and the Challenge of CG Face Differentiation}

Specialized neural structures focus on faces, and rely on affective information to fully process face stimuli. But what about stimuli that deviate from normal ones, particularly CG faces? While such questions are in their infancy, we receive telling insights from two phenomena where normal face processing is disrupted: the uncanny valley and pareidolia. In the uncanny valley phenomenon, human-like depictions elicit feelings of unease; stimuli meant to be faces are rejected. Conversely, in pareidolia, we perceive faces in inanimate objects, accepting non-face objects as faces. Together, these situations reveal insights about the boundaries of visual perception, offering hints of how we demarcate the visual world.
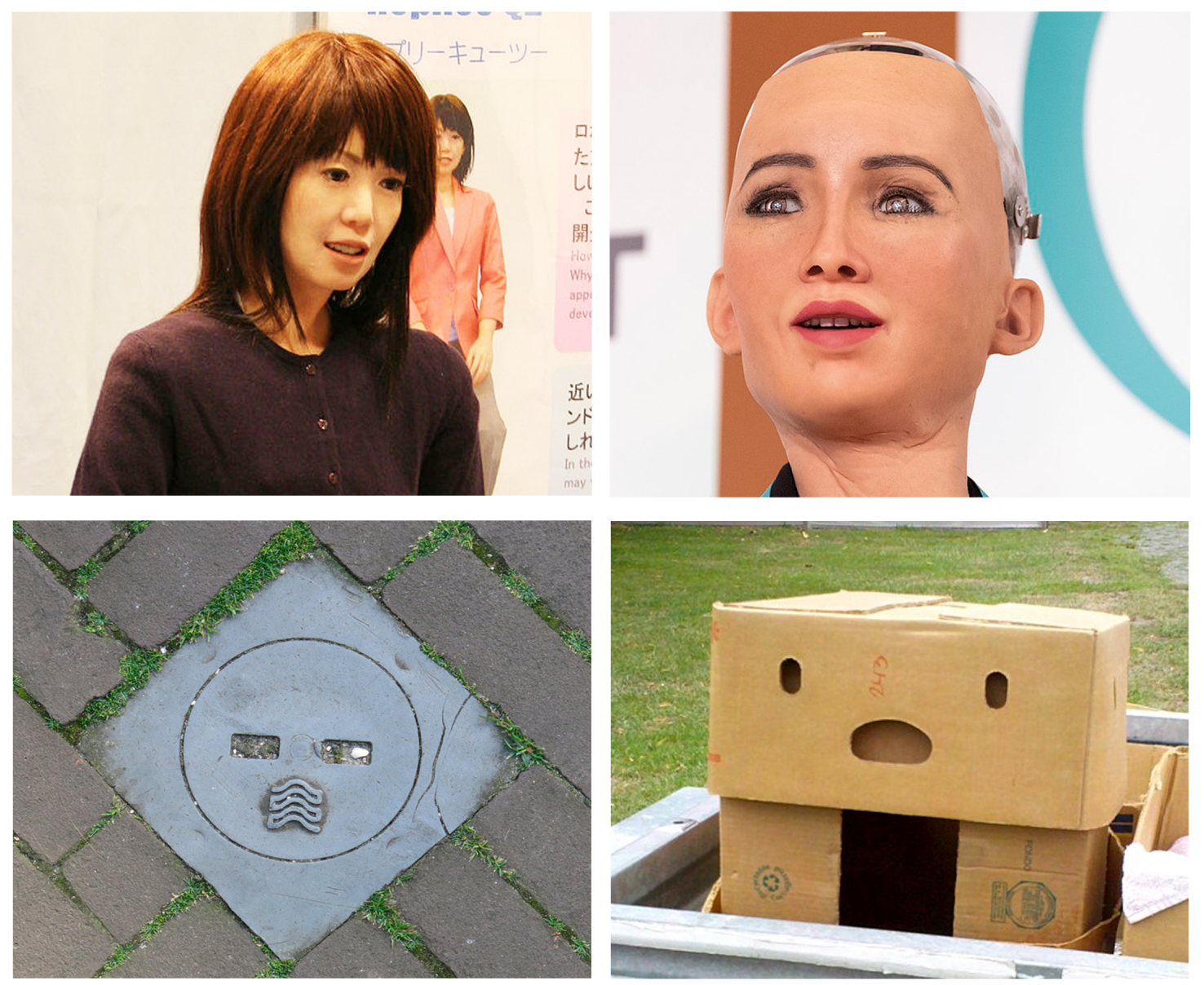

Figure 2. Examples of the uncanny valley effect (top) and pareidolia (bottom). Top left: Image copyright Brad Beattie. Top right: Image copyright UNCTAD. Bottom left: Image copyright Filip Maljković. Bottom right: Image copyright Alexander Gee. All retrieved from Wikimedia Commons under CC-BY-SA. 
In 1970, Japanese roboticist Masahiro Mori first described how humanoid robots can trigger mental unease. He compared the affinity humans felt for industrial robots (which lack faces) and toy robots (which appear more humanlike with a face). Humans felt a stronger affinity for toy robots, indicating a linear correlation between affinity and human likeness. Mori then extended this discussion to perceptions of prosthetic limbs and noticed how a highly humanlike hand can result in a feeling of creepiness - the previous linear correlation was disrupted (Mori et al., 2012). That is, rather than viewing humanlike depictions with empathy, our reaction was instead revulsion and discomfort, a feeling Mori named the "uncanny valley" (Mori et al., 2012). This was unexpected—as something resembled a human too closely, it elicited more unease. He published his results in 1970, noting this "descent into eeriness," but the idea did not gain widespread attention until 2005, when it was translated into English. (Mori et al., 2012, Lay, 2015).

Researchers have posited several theories for the uncanny valley, but there remains little consensus on what causes this phenomenon. It may occur because humans are unable to distinguish if what they are seeing is alive or not (Lundmark, 2017). Mori proposed that this feeling originally helped humans discriminate from corpses and members of different species (Mori et al., 2012). Other studies have investigated face characteristics to determine what features contribute to the uncanny valley effect, but the evidence has been inconsistent (Kätsyri et al., 2015). They found that atypical facial proportions, particularly a mismatch in eye and face size, were key factors in causing the phenomenon; other research shows that the uncanny valley effect emerges when there is an abnormal facial feature in addition to a highly realistic image (Seyama \& Nagayama, 2007). Individual differences, based on sociocultural constructions and biological adaptations for threat avoidance, also play a role in uncanny valley sensitivity (MacDorman \& Entezari, 2015). This has implications for the human-computer interaction space, as well as virtual reality and animation-it is important for designers and scientists to recognize these characteristics to create animations that don't disturb viewers (MacDorman et al., 2009). 


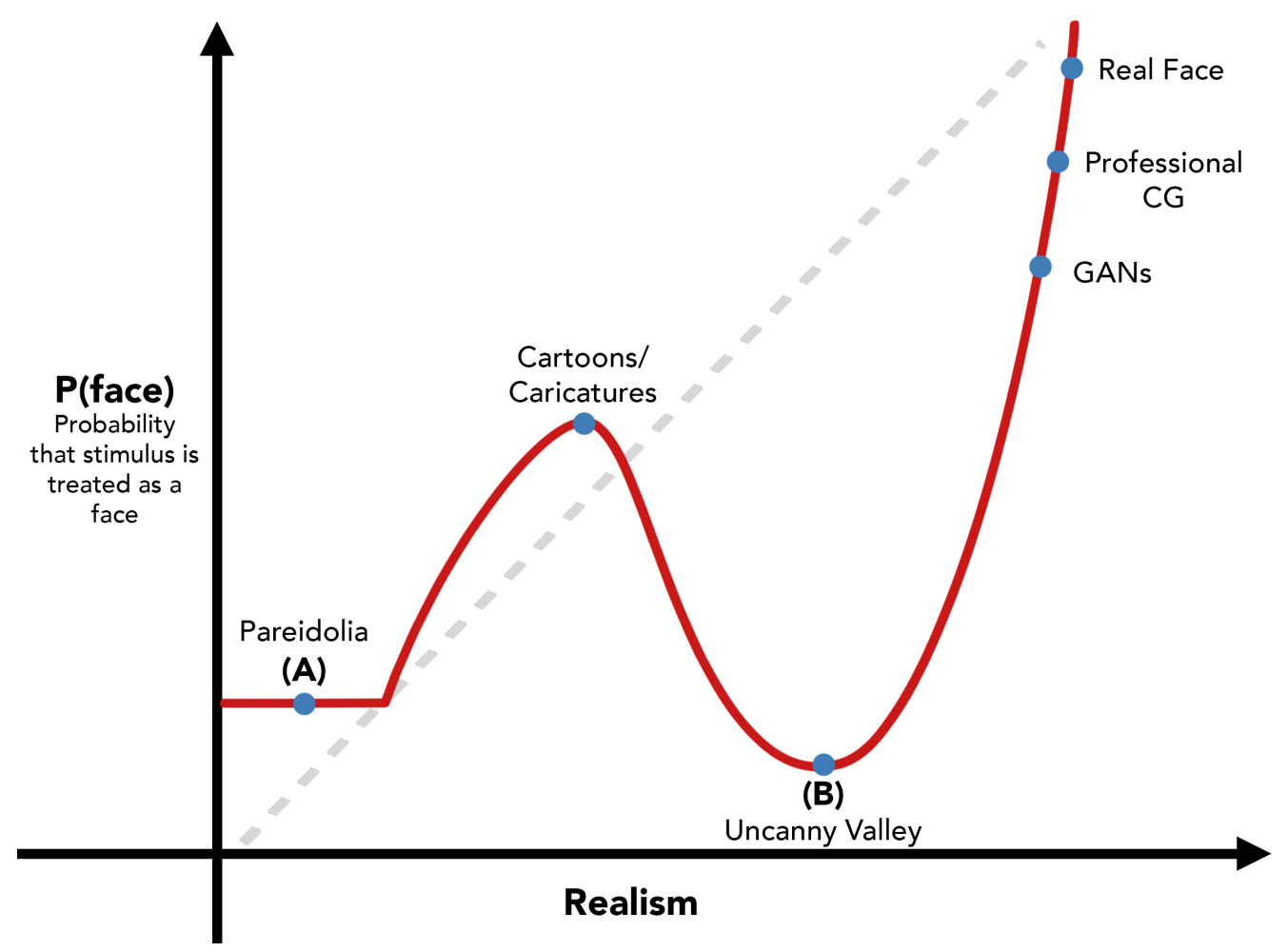

Figure 3. Schematic of the relationship between the realism of a stimulus and its perception as a face. Rather than being perceived as faces in proportion to their realism (gray line), face-like stimuli have a nonlinear perceptual relationship with realism (red line). In general, as realism increases, people are more likely to correctly react to the face. However, in pareidolia, behavioral responses are higher than expected on the basis of realism (A), while in the uncanny valley effect, responses are much lower $(B)$.

Conversely, if the uncanny valley is a Type 2 error in face recognition (a false negative), then pareidolia is a Type 1 error (a false positive), in which non-face stimuli are perceived as faces. Face pareidolia describes the illusion of seeing a face in another object, such as the moon or an electric outlet. The right fusiform face area is involved in viewing these illusory faces, and humans can even detect faces in images with pure noise (J. Liu et al., 2014). Pareidolia faces trigger a gaze-cuing attentional process only observed with face stimuli (Takahashi \& Watanabe, 2013). In fact, some computer animators and artists intentionally use pareidolia for artistic representations, as eye tracking patterns with face-like objects are very similar to those from faces (Making Faces: At the Intersection of Art and Neuroscience, 2016). Animators also create humanoid objects by eliciting subconscious pareidolia, which can help avoid uncanny valley effects (Lundmark, 2017). The uncanny valley and pareidolia tell us about the boundaries of where face processing systems operate. It is unknown whether processes involved in these phenomena are responsible for judging between real and CG faces, but they demonstrate that the acceptance regions for perceiving a stimulus as a face are larger than previously thought.

As to the specific processes that might allow us to distinguish between real and CG faces, an increasing number of studies have tackled this question directly. One study found the medial prefrontal cortex was involved in the perception of real humans whereas the superior parietal lobes 
were more involved with virtual-worlds, such as cartoon images (Han et al., 2005). Another study demonstrated that real faces evoked stronger fusiform face area and amygdala responses compared to CG ones (Kätsyri et al., 2019). These neurobiological differences between CG and real face processing suggest humans have some ability to discriminate between the two.

Other research has directly identified factors that allow for successful classification. One study displayed two faces (one real and one CG) to participants and asked them to label each (Fan et al., 2012). While image properties were important, the cognitive characteristics of viewers also impacted visual realism: laypersons and experts differed in their discrimination of CG and real faces. In comparison to color, shading of faces was more critical for visual realism (Fan et al., 2012). While some studies claim CG faces are processed in the same manner as face photographs (Matheson \& McMullen, 2011), others suggest that there are differences when directly comparing CG images with photographs (Crookes et al., 2015). Two established indicators of face expertise, identification of own-race faces and the other-race effect, were reduced for CG images (Crookes et al., 2015).

Cognitive illusions such as the uncanny valley and pareidolia give us insight into face perception and how we judge visual realities, but future research is needed to further understand how neural systems perceive and judge CG face stimuli, and whether it is a top-down or bottom-up process.

\section{Conclusion}

In the fields of politics, criminal justice, and more, members of the public are now increasingly called on to distinguish between real and CG faces. Thus, scientists are motivated to understand the basis of how humans perform this differentiation. Facial judgments involve highly specialized neural structures, occur rapidly, and bind visual and affective components for accurate identification and processing. Phenomena like the uncanny valley and pareidolia show paradoxes in processing and come with affective correlates. Some initial research offers insight as to how humans differentiate between real and CG faces. Real faces activate slightly different brain regions in comparison to CG images, and factors such as color and shading contribute to visual realism.

However, several unanswered questions remain regarding these face-related judgments. For example, it is unknown how exactly we merge representations of faces and affect, though studies show the importance of both factors. More research is also needed to understand which brain regions and processes are used to differentiate between real and CG faces, and whether this ability can be improved with training. Furthermore, little is known about the neurobiological correlates of paredolia. Exploring these research questions in the future will offer more insight into facial judgments.

In principle, understanding more about how humans differentiate between real and CG faces could be used to train people to identify differences. While there are automated methods to differentiate, many evaluations require people to perform this judgment, such as when jurors view photos as evidence in a criminal trial or voters are inundated with altered photos of politicians. In the future, additional research is needed to illuminate which brain regions are responsible and to 
develop training methods which utilize neuroscience and psychology insights to improve face-related judgments.

\section{Acknowledgments}

I would like to thank my faculty advisor Dr. John Pearson for his support and guidance during this research project. He provided important feedback regarding the scope of my research and literature review organization. I am also grateful for the support of the Duke University Psychology and Neuroscience $(P \& N)$ Department through a P\&N Small Grant. 


\section{$\underline{\text { References }}$}

Blais, C., Jack, R. E., Scheepers, C., Fiset, D., \& Caldara, R. (2008). Culture Shapes How We Look at Faces. PLOS ONE, 3(8), e3022. https://doi.org/10.1371/journal.pone.0003022

Calvo, M. G., Gutiérrez-García, A., Avero, P., \& Lundqvist, D. (2013). Attentional mechanisms in judging genuine and fake smiles: Eye-movement patterns. Emotion (Washington, D.C.), 13(4), 792-802. https://doi.org/10.1037/a0032317

Chhaya, T. (2017). She's Not My Mother: A 24-Year-Old Man With Capgras Delusion. https://www.mdedge.com/fedprac/article/153379/mental-health/shes-not-my-mother-24year-old-man-capgras-delusion

Collins, J. A., \& Olson, I. R. (2014). Beyond the FFA: The role of the ventral anterior temporal lobes in face processing. Neuropsychologia, 61, 65-79.

https://doi.org/10.1016/j.neuropsychologia.2014.06.005

Crookes, K., Ewing, L., Gildenhuys, J., Kloth, N., Hayward, W. G., Oxner, M., Pond, S., \& Rhodes, G. (2015). How Well Do Computer-Generated Faces Tap Face Expertise? PLOS ONE, 1O(11), e0141353. https://doi.org/10.1371/journal.pone.0141353

Dang-Nguyen, D.-T., Boato, G., \& De Natale, F. G. B. (2012). Discrimination between computer generated and natural human faces based on asymmetry information. 2012 Proceedings of the 20th European Signal Processing Conference (EUSIPCO), 1234-1238.

Dobs, K., Isik, L., Pantazis, D., \& Kanwisher, N. (2019). How face perception unfolds over time. Nature Communications, 10(1), 1-10. https://doi.org/10.1038/s41467-019-09239-1 
Eisenbarth, H., \& Alpers, G. W. (2011). Happy mouth and sad eyes: Scanning emotional facial expressions. Emotion (Washington, D.C.), 11(4), 860-865. https://doi.org/10.1037/a0022758

Ellis, H. D., Young, A. W., Quayle, A. H., \& De Pauw, K. W. (1997). Reduced autonomic responses to faces in Capgras delusion. Proceedings of the Royal Society of London. Series B: Biological Sciences, 264(1384), 1085-1092. https://doi.org/10.1098/rspb.1997.0150

Fan, S., Ng, T.-T., Herberg, J. S., Koenig, B. L., \& Xin, S. (2012). Real or Fake?: Human Judgments About Photographs and Computer-generated Images of Faces. SIGGRAPH Asia 2012 Technical Briefs, 17:1-17:4. https://doi.org/10.1145/2407746.2407763

Farid, H. (2006). Digital doctoring: How to tell the real from the fake. Significance, 3(4), 162-166. https://doi.org/10.1111/j.1740-9713.2006.00197.x

Gatys, L. A., Ecker, A. S., \& Bethge, M. (2016). Image Style Transfer Using Convolutional Neural Networks. 2016 IEEE Conference on Computer Vision and Pattern Recognition (CVPR), 2414-2423. https://doi.org/10.1109/CVPR.2016.265

Golarai, G., Liberman, A., \& Grill-Spector, K. (2017). Experience Shapes the Development of Neural Substrates of Face Processing in Human Ventral Temporal Cortex. Cerebral Cortex, 27(2). https://doi.org/10.1093/cercor/bhv314

Goodfellow, I., Pouget-Abadie, J., Mirza, M., Xu, B., Warde-Farley, D., Ozair, S., Courville, A., \& Bengio, Y. (2014). Generative Adversarial Nets. In Z. Ghahramani, M. Welling, C. Cortes, N. D. Lawrence, \& K. Q. Weinberger (Eds.), Advances in Neural Information Processing Systems 27 (pp. 2672-2680). Curran Associates, Inc. http://papers.nips.cc/paper/5423generative-adversarial-nets.pdf 
Gross, C. G. (2002). Genealogy of the "Grandmother Cell.” The Neuroscientist, 8(5), 512-518. https://doi.org/10.1177/107385802237175

Gross, C. G., De Schonen, S., Bruce, V., Cowey, A., Ellis, A. W., \& Perrett, D. I. (1992). Representation of visual stimuli in inferior temporal cortex. Philosophical Transactions of the Royal Society of London. Series B: Biological Sciences, 335(1273), 3-10.

https://doi.org/10.1098/rstb.1992.0001

Gunning-Dixon, F. M., Gur, R. C., Perkins, A. C., Schroeder, L., Turner, T., Turetsky, B. I., Chan, R. M., Loughead, J. W., Alsop, D. C., Maldjian, J., \& Gur, R. E. (2003). Age-related differences in brain activation during emotional face processing. Neurobiology of Aging, 24(2), 285-295. https://doi.org/10.1016/S0197-4580(02)00099-4

Han, S., Jiang, Y., Humphreys, G. W., Zhou, T., \& Cai, P. (2005). Distinct neural substrates for the perception of real and virtual visual worlds. NeuroImage, 24(3), 928-935. https://doi.org/10.1016/j.neuroimage.2004.09.046

Harris, L. T., \& Fiske, S. T. (2006). Dehumanizing the Lowest of the Low: Neuroimaging Responses to Extreme Out-Groups. Psychological Science, 17(10), 847-853. https://doi.org/10.1111/j.1467-9280.2006.01793.x

Harris, L. T., \& Fiske, S. T. (2007). Social groups that elicit disgust are differentially processed in mPFC. Social Cognitive and Affective Neuroscience, 2(1), 45-51. https://doi.org/10.1093/scan/ns1037

Hirstein, W., \& Ramachandran, V. S. (1997). Capgras syndrome: A novel probe for understanding the neural representation of the identity and familiarity of persons. Proceedings of the Royal 
Society of London. Series B: Biological Sciences, 264(1380), 437-444.

https://doi.org/10.1098/rspb.1997.0062

Jiang, F., Blanz, V., \& Rossion, B. (2011). Holistic processing of shape cues in face identification:

Evidence from face inversion, composite faces, and acquired prosopagnosia. Visual Cognition, 19(8), 1003-1034. https://doi.org/10.1080/13506285.2011.604360

Jiang, L., Wu, W., Li, R., Qian, C., \& Loy, C. C. (2020). DeeperForensics-1.0: A Large-Scale Dataset for Real-World Face Forgery Detection. 17.

Johnson, M. H., Dziurawiec, S., Ellis, H., \& Morton, J. (1991). Newborns' preferential tracking of face-like stimuli and its subsequent decline. Cognition, 40(1), 1-19. https://doi.org/10.1016/0010-0277(91)90045-6

Kanwisher, N., McDermott, J., \& Chun, M. M. (1997). The Fusiform Face Area: A Module in Human Extrastriate Cortex Specialized for Face Perception. Journal of Neuroscience, 17(11), 4302-4311. https://doi.org/10.1523/JNEUROSCI.17-11-04302.1997

Kätsyri, J., de Gelder, B., \& de Borst, A. W. (2019). Amygdala responds to direct gaze in real but not in computer-generated faces. NeuroImage, 204, 116216. https://doi.org/10.1016/j.neuroimage.2019.116216

Kätsyri, J., Förger, K., Mäkäräinen, M., \& Takala, T. (2015). A review of empirical evidence on different uncanny valley hypotheses: Support for perceptual mismatch as one road to the valley of eeriness. Frontiers in Psychology, 6. https://doi.org/10.3389/fpsyg.2015.00390

Kelly, D. J., Liu, S., Rodger, H., Miellet, S., Ge, L., \& Caldara, R. (2011). Developing cultural differences in face processing. Developmental Science, 14(5), 1176-1184. https://doi.org/10.1111/j.1467-7687.2011.01067.x 
Korshunov, P., \& Marcel, S. (2019). Vulnerability of Face Recognition to Deep Morphing. ArXiv:1910.01933 [Cs]. http://arxiv.org/abs/1910.01933

Kreiman, G., Koch, C., \& Fried, I. (2000). Category-specific visual responses of single neurons in the human medial temporal lobe. Nature Neuroscience, 3(9), 946-953. https://doi.org/10.1038/78868

Kriegeskorte, N., Formisano, E., Sorger, B., \& Goebel, R. (2007). Individual faces elicit distinct response patterns in human anterior temporal cortex. Proceedings of the National Academy of Sciences of the United States of America, 104(51), 20600-20605. https://doi.org/10.1073/pnas.0705654104

Lay, S. (2015, November 13). Uncanny valley: Why we find human-like robots and dolls so creepy | Stephanie Lay. The Guardian. https://www.theguardian.com/commentisfree/2015/nov/13/robots-human-uncanny-valley

Liu, J., Li, J., Feng, L., Li, L., Tian, J., \& Lee, K. (2014). Seeing Jesus in toast: Neural and behavioral correlates of face pareidolia. Cortex, 53, 60-77. https://doi.org/10.1016/j.cortex.2014.01.013

Liu, S., Quinn, P. C., Wheeler, A., Xiao, N., Ge, L., \& Lee, K. (2011). Similarity and difference in the processing of same- and other-race faces as revealed by eye tracking in 4- to 9-montholds. Journal of Experimental Child Psychology, 108(1), 180-189. https://doi.org/10.1016/j.jecp.2010.06.008

Lundmark, M. (2017). How to breath life into inanimate objects. http://urn.kb.se/resolve?urn=urn:nbn:se:ltu:diva-63852 
MacDorman, K. F., \& Entezari, S. O. (2015). Individual differences predict sensitivity to the uncanny valley. Interaction Studies, 16(2), 141-172. https://doi.org/10.1075/is.16.2.01mac

MacDorman, K. F., Green, R. D., Ho, C.-C., \& Koch, C. T. (2009). Too real for comfort? Uncanny responses to computer generated faces. Computers in Human Behavior, 25(3), 695710. https://doi.org/10.1016/j.chb.2008.12.026

Making Faces: At the Intersection of Art and Neuroscience. (2016). Retrieved October 26, 2019, from https://nasher.duke.edu/large-files/pdfs/making-faces.pdf

Mapping the Social Space of the Face. (n.d.). Https://Www.Apa.Org. Retrieved October 8, 2019, from https://www.apa.org/science/about/psa/2010/03/sci-brief

Matheson, H. E., \& McMullen, P. A. (2011). A computer-generated face database with ratings on realism, masculinity, race, and stereotypy. Behavior Research Methods, 43(1), 224-228. https://doi.org/10.3758/s13428-010-0029-9

Moeller, S., Freiwald, W. A., \& Tsao, D. Y. (2008). Patches with Links: A Unified System for Processing Faces in the Macaque Temporal Lobe. Science, 320(5881), 1355-1359. https://doi.org/10.1126/science. 1157436

Mori, M., MacDorman, K. F., \& Kageki, N. (2012). The Uncanny Valley [From the Field]. IEEE Robotics Automation Magazine, 19(2), 98-100. https://doi.org/10.1109/MRA.2012.2192811

Mormann, F., Kornblith, S., Quiroga, R. Q., Kraskov, A., Cerf, M., Fried, I., \& Koch, C. (2008). Latency and Selectivity of Single Neurons Indicate Hierarchical Processing in the Human Medial Temporal Lobe. Journal of Neuroscience, 28(36), 8865-8872. https://doi.org/10.1523/JNEUROSCI.1640-08.2008 
Mukhopadhyay, D., Shirvanian, M., \& Saxena, N. (2015). All Your Voices are Belong to Us: Stealing Voices to Fool Humans and Machines. In G. Pernul, P. Y A Ryan, \& E. Weippl (Eds.), Computer Security—ESORICS 2015 (pp. 599-621). Springer International Publishing. https://doi.org/10.1007/978-3-319-24177-7_30

Nemrodov, D., Niemeier, M., Mok, J. N. Y., \& Nestor, A. (2016). The time course of individual face recognition: A pattern analysis of ERP signals. NeuroImage, 132, 469-476. https://doi.org/10.1016/j.neuroimage.2016.03.006

Nestor, A., Plaut, D. C., \& Behrmann, M. (2011). Unraveling the distributed neural code of facial identity through spatiotemporal pattern analysis. Proceedings of the National Academy of Sciences, 108(24), 9998-10003. https://doi.org/10.1073/pnas.1102433108

Proverbio, A. M., Brignone, V., Matarazzo, S., Del Zotto, M., \& Zani, A. (2006). Gender differences in hemispheric asymmetry for face processing. BMC Neuroscience, 7(1), 44. https://doi.org/10.1186/1471-2202-7-44

Quiroga, R. Q., Reddy, L., Kreiman, G., Koch, C., \& Fried, I. (2005). Invariant visual representation by single neurons in the human brain. Nature, 435(7045), 1102-1107. https://doi.org/10.1038/nature03687

Riesenhuber, M., Jarudi, I., Gilad, S., \& Sinha, P. (2004). Face processing in humans is compatible with a simple shape-based model of vision. Proceedings of the Royal Society of London. Series B: Biological Sciences, 271(suppl_6). https://doi.org/10.1098/rsbl.2004.0216

Rössler, A., Cozzolino, D., Verdoliva, L., Riess, C., Thies, J., \& Nießner, M. (2018). FaceForensics: A Large-scale Video Dataset for Forgery Detection in Human Faces. ArXiv:1803.09179 [Cs]. http://arxiv.org/abs/1803.09179 
Rutishauser, U., Tudusciuc, O., Neumann, D., Mamelak, A. N., Heller, A. C., Ross, I. B., Philpott, L., Sutherling, W. W., \& Adolphs, R. (2011). Single-Unit Responses Selective for Whole Faces in the Human Amygdala. Current Biology, 21(19), 1654-1660. https://doi.org/10.1016/j.cub.2011.08.035

Schirmer, A., \& Adolphs, R. (2017). Emotion Perception from Face, Voice, and Touch: Comparisons and Convergence. Trends in Cognitive Sciences, 21(3), 216-228. https://doi.org/10.1016/j.tics.2017.01.001

Schrouff, J., Raccah, O., Baek, S., Rangarajan, V., Salehi, S., Mourão-Miranda, J., Helili, Z., Daitch, A. L., \& Parvizi, J. (2020). Fast temporal dynamics and causal relevance of face processing in the human temporal cortex. Nature Communications, 11(1), 1-13. https://doi.org/10.1038/s41467-020-14432-8

Schurgin, M. W., Nelson, J., Iida, S., Ohira, H., Chiao, J. Y., \& Franconeri, S. L. (2014). Eye movements during emotion recognition in faces. Journal of Vision, 14(13), 14. https://doi.org/10.1167/14.13.14

Seyama, J., \& Nagayama, R. S. (2007). The Uncanny Valley: Effect of Realism on the Impression of Artificial Human Faces. Presence: Teleoperators and Virtual Environments, 16(4), 337-351. https://doi.org/10.1162/pres.16.4.337

Takahashi, K., \& Watanabe, K. (2013). Gaze Cueing by Pareidolia Faces. I-Perception, 4(8), 490492. https://doi.org/10.1068/i0617sas

Thomas, L. A., Bellis, M. D. D., Graham, R., \& LaBar, K. S. (2007). Development of emotional facial recognition in late childhood and adolescence. Developmental Science, 10(5), 547-558. https://doi.org/10.1111/j.1467-7687.2007.00614.x 
Tsao, D. Y., Freiwald, W. A., Tootell, R. B. H., \& Livingstone, M. S. (2006). A Cortical Region Consisting Entirely of Face-Selective Cells. Science (New York, N.Y.), 311(5761), 670-674. https://doi.org/10.1126/science.1119983

Tsao, D. Y., Moeller, S., \& Freiwald, W. A. (2008). Comparing face patch systems in macaques and humans. Proceedings of the National Academy of Sciences, 105(49), 19514-19519. https://doi.org/10.1073/pnas.0809662105

Venkataramakrishnan, S. (2019, October 24). Can you believe your eyes? How deepfakes are coming for politics. Financial Times. https://www.ft.com/content/4bf4277c-f527-11e9-a79cbc9acae3b654

Vuilleumier, P., \& Pourtois, G. (2007). Distributed and interactive brain mechanisms during emotion face perception: Evidence from functional neuroimaging. Neuropsychologia, 45(1), 174-194. https://doi.org/10.1016/j.neuropsychologia.2006.06.003

Wang, W. Y. (2017). "Liar, Liar Pants on Fire”: A New Benchmark Dataset for Fake News Detection. ArXiv:1705.00648 [Cs]. http://arxiv.org/abs/1705.00648

Wang, Y., Metoki, A., Smith, D. V., Medaglia, J. D., Zang, Y., Benear, S., Popal, H., Lin, Y., \& Olson, I. R. (2020). Multimodal mapping of the face connectome. Nature Human Behaviour, 4(4), 397-411. https://doi.org/10.1038/s41562-019-0811-3

Webster, M. A., \& MacLeod, D. I. A. (2011). Visual adaptation and face perception. Philosophical Transactions of the Royal Society B: Biological Sciences, 366(1571), 1702-1725. https://doi.org/10.1098/rstb.2010.0360 
Wells, L. J., Gillespie, S. M., \& Rotshtein, P. (2016). Identification of Emotional Facial Expressions: Effects of Expression, Intensity, and Sex on Eye Gaze. PLoS ONE, 11(12). https://doi.org/10.1371/journal.pone.0168307

Willis, J., \& Todorov, A. (2006). First Impressions: Making Up Your Mind After a 100-Ms Exposure to a Face. Psychological Science, 17(7), 592-598. https://doi.org/10.1111/j.14679280.2006.01750.x

Yarkoni, T., Poldrack, R. A., Nichols, T. E., Van Essen, D. C., \& Wager, T. D. (2011). Large-scale automated synthesis of human functional neuroimaging data. Nature Methods, 8(8), 665670. https://doi.org/10.1038/nmeth.1635 